\title{
Design and performance analysis of a PIAACMC coronagraph on a segmented aperture
}

Ruslan Belikov, Stephen Bryson, Dan Sirbu, Olivier Guyon, Eduardo Bendek, et al.

Ruslan Belikov, Stephen Bryson, Dan Sirbu, Olivier Guyon, Eduardo Bendek, Brian Kern, "Design and performance analysis of a PIAACMC coronagraph on a segmented aperture," Proc. SPIE 10698, Space Telescopes and Instrumentation 2018: Optical, Infrared, and Millimeter Wave, 106981H (1 August 2018); doi: 10.1117/12.2314202

Event: SPIE Astronomical Telescopes + Instrumentation, 2018, Austin, Texas, United States 


\title{
Design and performance analysis of a PIAACMC coronagraph on a segmented aperture
}

\author{
Ruslan Belikov ${ }^{*}$, Stephen Bryson ${ }^{\mathrm{a}}$, Dan Sirbu ${ }^{\mathrm{a}}$, Olivier Guyon ${ }^{\mathrm{b}}$, Eduardo Bendek ${ }^{\mathrm{a}}$, Brian Kern ${ }^{\mathrm{c}}$, \\ ${ }^{a}$ NASA Ames Research Center, Moffett Field \\ ${ }^{\mathrm{b}}$ The University of Arizona \\ ${ }^{\mathrm{N}}$ NASA Jet Propulsion Laboratory
}

\begin{abstract}
To directly image and characterize exoplanets, starlight suppression systems rely on coronagraphs to optically remove starlight while preserving planet light for spectroscopy. The Phase-Induced Amplitude Apodization Complex Mask Coronagraph (PIAACMC) is an attractive coronagraph option for the next generation of large space telescopes optimized for habitable exoplanet imaging: PIAACMC offers high throughput, small inner working angle (IWA) with little loss in image quality. PIAACMC is also compatible with segmented apertures, preserving much of the throughput and resolution of a full pupil. Coronagraph compatibility with segmented apertures is essential for the success of habitable planet characterization with future large apertures, such as the Large UV / Optical/ Infrared (LUVOIR) concept currently under way to inform the 2020 decadal survey.

We present a design of PIAACMC for a segmented aperture, using the segmented aperture currently considered for LUVOIR as a representative case. This design is optimized to be resilient to tip/tilt jitter and large stellar angular sizes. This also enables it to have improved tolerance to polarization-specific aberrations, which are dominated by low-order modes such as tip-tilt and astigmatism. We simulate and study the performance of this design using a simplified instrument model. These simulations include wavefront control and tip-tilt errors. We characterize the performance of our design in monochromatic as well as broadband light in terms of throughput, inner working angle, contrast, area of the dark zone, and sensitivity to low-order aberrations.
\end{abstract}

Keywords: coronagraph, high contrast, LUVOIR, exoplanet, direct imaging, PIAA, PIAACMC, habitable

\section{PIAACMC OVERVIEW AND MOTIVATION}

To directly image and characterize exoplanets, starlight suppression systems rely on coronagraphs to optically remove starlight while preserving planet light for spectroscopy. PIAACMC, or Phase-Induced Amplitude Apodization Complex Mask Coronagraph [1] is a high performance coronagraph capable of close to $100 \%$ coronagraphic throughput, and inner working angles (IWAs) that can go as low as $0.5 \lambda / \mathrm{D}$. Its sensitivity to tip/tilt aberrations is close to theoretical limits at least for aggressive inner working angles [2]. Thus, PIAACMC represents a path to reach physics-limited (rather than coronagraph-architecture-limited) starlight suppression performance for a given aperture and stability environment. This path can enable taking full advantage of the large apertures on future telescopes like LUVOIR and HabEx, and maximize their science and cost effectiveness.

In certain key respects, the performance gains enabled by high performance coronagraphs are equivalent to increasing the aperture of the telescope. For example, a PIAACMC coronagraph with $80 \%$ throughput and an inner working angle of 1.5 $\lambda / \mathrm{D}$ on an $4 \mathrm{~m}$ aperture is equivalent to an $8 \mathrm{~m}$ telescope that has a coronagraph with a $20 \%$ throughput and $3 \lambda / \mathrm{D}$, in terms of photon flux and inner working angle in units of arcseconds. Conversely, missions can save significant costs by reducing the aperture and improving coronagraph performance, leaving the science yield unchanged.

* ruslan.belikov@nas.gov; $\quad$ phone 1-650-604-0833

Space Telescopes and Instrumentation 2018: Optical, Infrared, and Millimeter Wave, edited by Makenzie Lystrup,

Howard A. MacEwen, Giovanni G. Fazio, Proc. of SPIE Vol. 10698, 106981H

(C) 2018 SPIE · CCC code: 0277-786X/18/\$18 - doi: 10.1117/12.2314202

Proc. of SPIE Vol. 10698 106981H-1 


\subsection{Science motivation}

The importance of enabling small IWAs is highlighted in [3]. In this study, the mission yield of Earth-like exoplanets in the habitable zones of their stars grew approximately inversely to IWA, meaning a mission with half the IWA doubles the yield of Earth-like exoplanets. Considering all exoplanets (not just Earth-like exoplanets), the yield grows as the inverse $3^{\text {rd }}$ power of IWA for a target-limited mission. Thus, advancing coronagraph architectures with small IWAs has a greater impact on expected yield than parameters such as increasing throughput or improving contrast.

A throughput improvement also increases yield via several ways. It leads to faster wavefront control loops (at least if the loop rate is photon-noise limited) and thus better correction of dynamic speckles, improving raw contrast. Higher photon fluxes also enable better post-processing (in the photon-noise limited regime), enabling deeper post-processed contrasts. Higher SNR and/or higher spectral resolution are enabled by higher throughput. For time-limited missions, throughput leads directly to higher yield even if nothing else is improved.

\subsection{Providing useful techniques to other coronagraph architectures}

In addition to being a stand-alone architecture, PIAACMC can be hybridized with other coronagraph architectures, which is sometimes necessary to make those architectures compatible with segmented apertures. For example, the Vortex coronagraph requires an apodization for segmented apertures, which can be implemented by a set of PIAA mirrors. Similarly, the apodization in the Apodized Pupil Lyot Coronagraph (APLC) can be implemented using PIAA mirrors, which improves throughput and inner working angle. The focal plane masks developed for PIAA (design tools as well as fabrication methods) may also be useful as alternative option for the Bandlimited Lyot and related coronagraphs. It may also be fruitful to explore the possibility of applying CMC-like features to the Vortex coronagraph mask, which may help with segmented apertures.

\subsection{Overview of PIAACMC optics and layout}

Phase Induced Amplitude Apodized Complex Mask Coronagraph (PIAACMC)

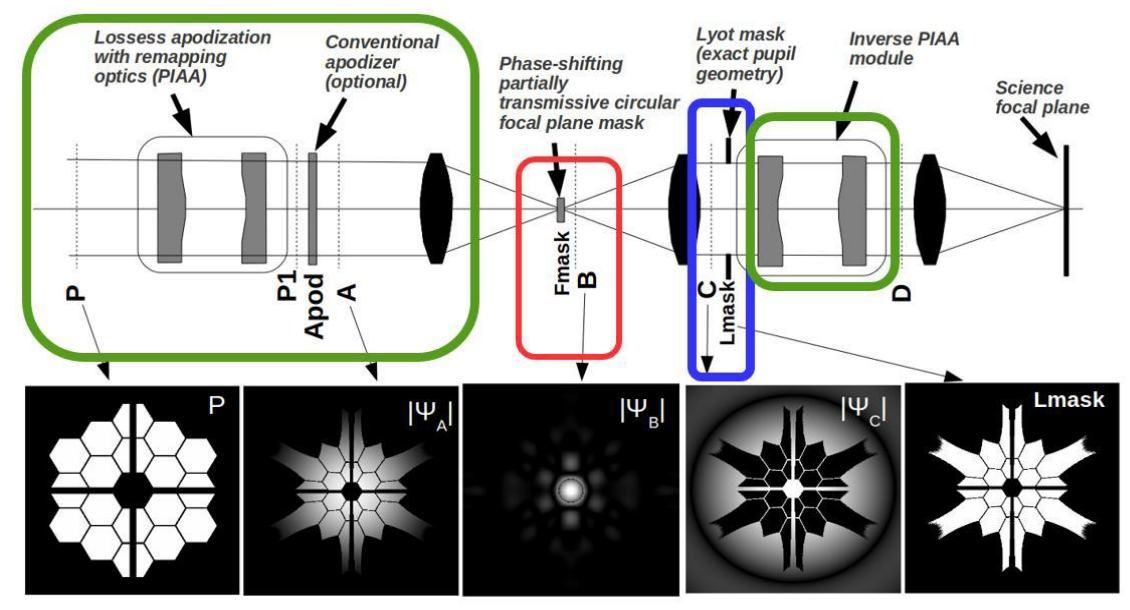

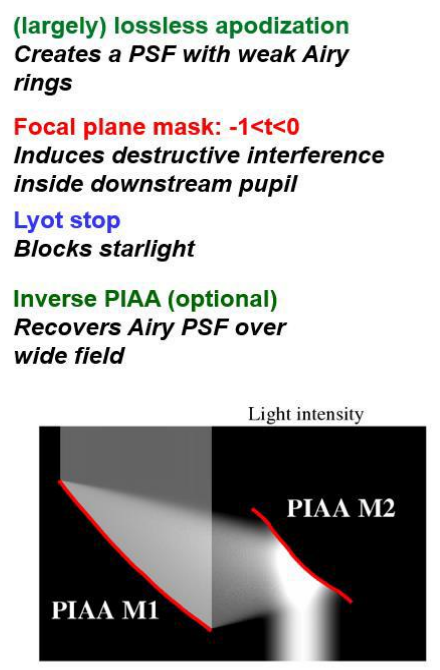

(largely) lossless apodization rings

Focal plane mask: $-1<\mathrm{t}<0$ Induces destructive interference inside downstream pupil

Inverse PIAA (optional) Recovers Airy PSF over wide field

Figure 1. Schematic diagram of PIAACMC coronagraph.

The PIAACMC coronagraph is diagrammed in Figure 1 and consists of:

- A pair of PIAA mirrors that remap the intensity profile of the incoming beam (P) from a top-hat shape into a specially designed apodization (A). For unobstructed apertures, this apodization has a so-called prolatespheroidal shape, but for obstructed apertures it becomes more complicated and needs to be numerically optimized.

- A phase-shifting partially transmissive focal plane mask. A pi-phase-shifting mask can cause complete destructive interference of light in the openings of the Lyot stop, if its transmission is chosen appropriately. The "ideal" pi-phase-shifting partially transmitting mask (over an entire wavelength bandpass) is challenging to manufacture directly, so practical PIAACMC designs use an array of fully reflective (or fully transmissive) 
zones, each with some specific height to generate some desired optical path difference for that zone. The zone heights are optimized to achieve the best contrast in a desired region of interest. CMC masks do not absorb light, rather they redirect on-axis starlight to the Lyot stop.

- A Lyot stop blocks starlight that is diffracted by the focal plane mask. In a simplistic treatment, the Lyot stop will be identical to the (remapped) segmented pupil obscuration, as shown in Figure 1. In practice, more area is blocked to accommodate real-world near-field diffraction and other practical concerns.

- An (optional) inverse PIAA optics is used to undo the pupil remapping, without which the field of view is limited by off-axis distortions caused by the PIAA optics. For many practical designs, including the WFIRST PIAACMC design, this is not necessary.

\section{PIAACMC DESIGN AND TESTING PIPELINE}

Our design and test pipeline is diagrammed in Figure 2. We used a "compact" layout where several components are assumed to be in the same plane and all components are assumed to be the same size. In reality, there will be reimaging optics between, for example, the pupil and PIAA1 mirror, or DM (Deformable Mirror) and PIAA2 mirror, and not all optics will be the same size. However, the compact design abstracts this away for simplicity, because these details usually do not affect the design of the coronagraph components themselves. A compact design can be converted into a real layout without changing design of components as long as the Fresnel number of each component stays the same.

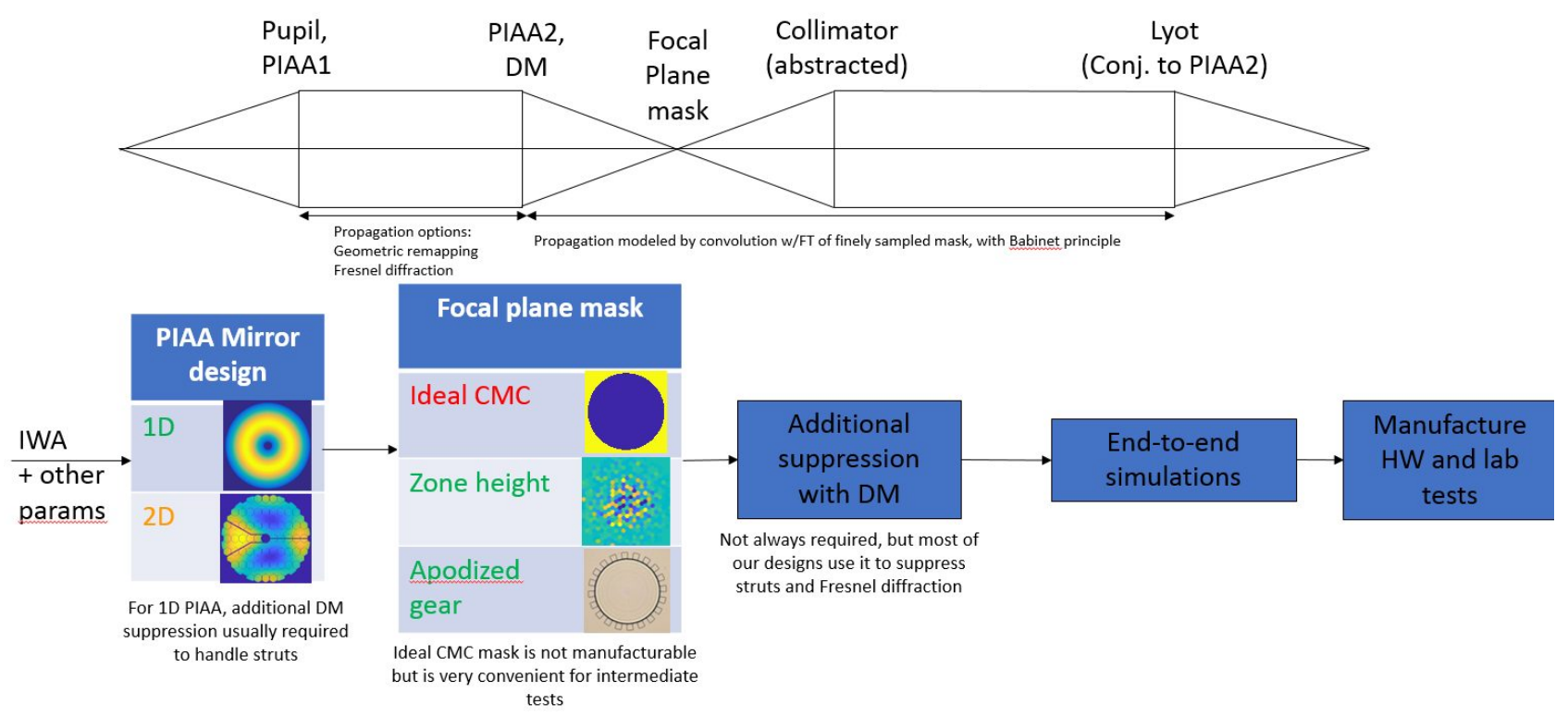

Figure 2. Design and testing pipeline. Top: simplified diagram of the PIAA layout used for designing PIAACMC components (PIAA mirrors, CMC mask, Lyot stop). Bottom: Design work flow. PIAA mirrors are designed first, then the focal plane mask, followed by additional suppression by the deformable mirror. The last two boxes (end-to-end simulations and manufacture $H W$ and lab tests are outside the scope of this presentation).

We used two methods to propagate between PIAA optics, depending on the approximation level and computational speed required for a particular task: simple geometrical remapping (i.e. the limit of infinitely small wavelengths, or ray optics), or Fresnel diffraction propagation (treating the PIAA optics as infinitely thin plates which impose an optical path delay that is a function of the lateral coordinates). The propagation between PIAA2+DM plane was implemented by a convolution between the electric field immediately after the PIAA+DM plane and the Fourier Transform of the Focal Plane mask. The focal plane mask itself in general has the form of some complex-valued transmission $M(x, y)$, or $M(r, \theta)$ in polar coordinates. Beyond some value $r>R_{\text {mask }}$, the mask is fully transmissive, i.e. $M(r, \theta)=1$. In order to numerically model such a mask with finite-sized arrays, we used a standard technique based on Babinet's principle, where instead of using the actual $M$ as our mask, we used $M^{\prime}=M-1$, and then applied a correction in the Lyot plane (adding the electric field corresponding to the case $M=1$ ). This allows representing the mask transmission by an array sampled on a grid going out only to $R_{\text {mask }}$, which saves significant memory and computational speed. 
We explored several design strategies for PIAA mirrors and focal plane mask. A key branching point is whether to design a coronagraph (PIAA optics and mask) for a 1D pupil, i.e. a pupil as a function of radius only, which includes the central obstruction but not segments or struts, or for a full 2D pupil. For the case of a coronagraph designed for a 1D pupil, additional suppression with a DM is required to suppress light leaked from struts and segments. As long as the struts and segments are sufficiently thin, this usually results in a realistic shape on the DM. It turns out that the LUVOIR pupil struts and segments are indeed sufficiently thin for the DM to suppress them (as will be shown in section 5), and so we focused our design efforts of the PIAA optics for a 1D pupil.

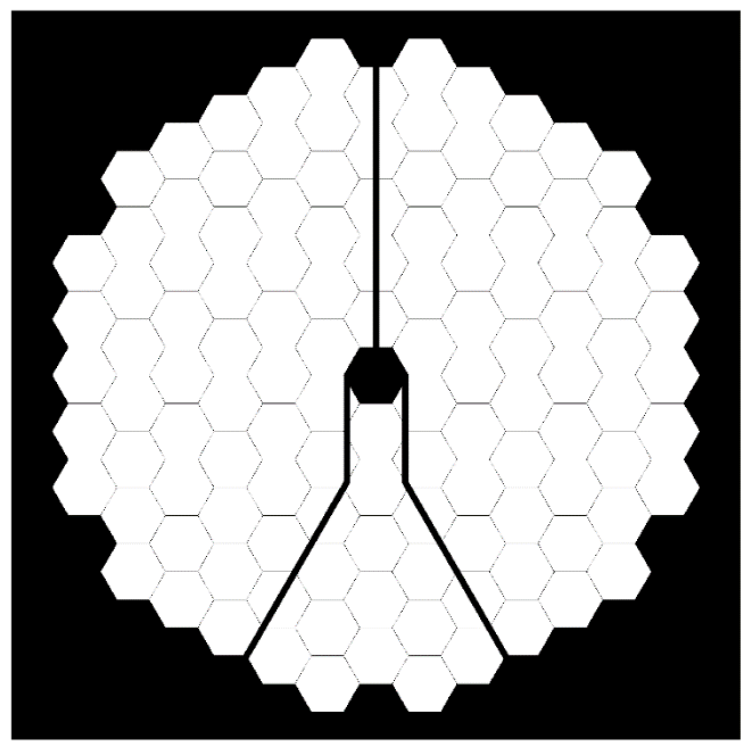

Figure 3. Representative segmented aperture used for this work: the LUVOIR pupil

After PIAA mirrors are designed, the next step is to design a focal plane mask, and we considered three options: an ideal "Complex-valued Mask for Coronagraphs" (ideal CMC) which achieves full coronagraphic suppression in broadband light [2] but is not manufacturable with current technologies; "zone height" masks which are manufacturable but are computationally intensive to design; and so-called "apodized gear" masks which are less computationally intensive to design but are less mature than the zone height masks. In this paper, we focus on a design based on the zone height masks, and will cover masks in more detail in Section 4.

After the PIAA mirrors and masks are designed for an obstructed 1D pupil, we insert the full 2D pupil (i.e. struts and segment gaps), and compute a surface on the deformable mirror that suppresses the light leak from struts and segment gaps, as described in section 5 .

Also shown in Figure 2 are on-going and future efforts outside the scope of this paper: end-to-end simulations as well as manufacturing and laboratory tests.

\section{PIAA MIRROR AND IDEAL PIAACMC DESIGN}

Our PIAA mirror design methods are based on $[1,4,5]$. In this step of the process, we essentially compute an ideal PIAACMC coronagraph [1] for the compact layout in Figure 2. An ideal PIAACMC involves two idealizations: (1) there is no diffraction propagation between the two PIAA mirrors, so that the propagation is purely geometric; (2) the mask is the "ideal CMC", which is a simple 2-zone mask, where the transmission is some value $-t$ inside mask radius $q_{0}$, and 1 
otherwise. The value of $t$ is some constant between 0 and 1 , depending on the mask radius $q_{0}$, so that the inside of the mask imposes an achromatic partially transmissive pi-phase shift. The Lyot stop in the ideal coronagraph is identical to the pupil.

Ideal PIAACMC coronagraphs achieve full coronagraphic suppression of on-axis sources for arbitrarily obstructed pupils in broadband light. For any given pupil apertures at PIAA1 and PIAA2 planes, there is a continuous family of PIAACMC designs parametrized by mask radius $q_{0}$, or equivalently the inner working angle of the coronagraph, which is monotonically related to $q_{0}$. This relationship is shown in Figure 4, for the case where the pupil apertures at PIAA1 and PIAA 2 are both circular apertures with a $10 \%$ obstruction in the middle. All designs in this section have this pair of apertures at PIAA1 and PIAA2. The inner working angle is defined as the off-axis angle of a point source in the sky, in $\lambda / \mathrm{D}$ units, at which the total energy transmitted to the final science plane is $1 / 2$ of the total energy immediately after the entrance pupil. Inner working angles can be as small as $\sim 0.5 \lambda / \mathrm{D}$, but larger inner working angles result in coronagraphs that are more tolerant to tip/tilt errors and angular stellar sizes. The qo parameter values are listed in "system $\lambda / \mathrm{D}$ units", which essentially treat PIAA2 plane as the pupil of the system instead of PIAA1. Hence, the relation between on-sky $\lambda / \mathrm{D}$ units and system $\lambda / \mathrm{D}$ units is the effective magnification induced by the two PIAA optics, because remapping between PIAA1 and PIAA2 changes the effective diameter of the pupil.

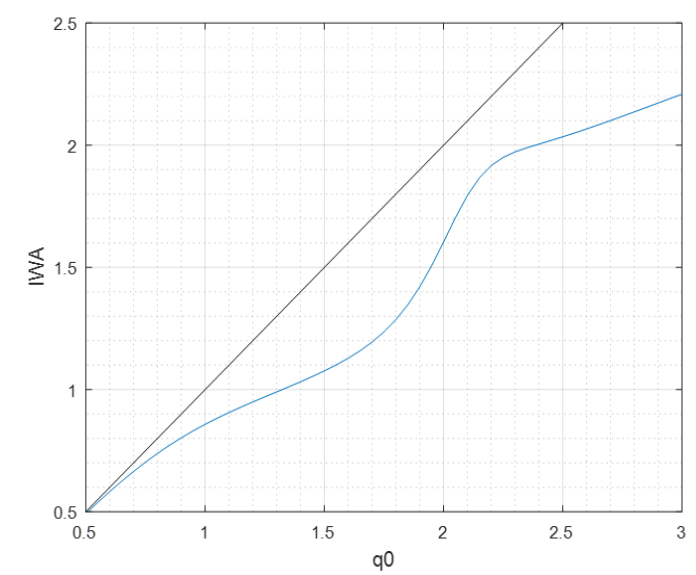

Figure 4. Relationship between the mask radius q0 (in l/D system units), and the inner working angle of the system, in blue color. The black line is $I W A=q 0$, shown simply for reference of no magnification.

In Figure 5, we show two selected points from the continuous family of PIAACMC designs, corresponding to $\mathrm{q}_{0}=2.6$ and 1.9 , respectively (inner working angles of 2.07 and $1.4 \lambda / \mathrm{D}$ ). As $\mathrm{q}_{0}$ increases, the apodization and PIAA shapes change from monotonic ones (such as the one on the bottom of Figure 5) where the only extrema are on the inner and outer edges of the aperture, to "donut" shapes, where there is an extremum between the aperture edges. Figure 5 also shows how the geometric propagation idealization (red curve) compares to Fresnel diffraction (blue curve), for the case of 36mm diameter optics separated by $40 \mathrm{~cm}$. Fresnel diffraction causes a contrast degradation from 0 (full suppression) to $\sim 1 \mathrm{e}-7$ in this case, which we note here but defer a more detailed discussion till section 5, where we show that a deformable mirror can be used to suppress residual light due to Fresnel diffraction.

We also studied the sensitivity of ideal PIAACMC designs to tip/tilt, as well as two different variations of the ideal PIAACMC focal plane mask. Ideal PIAACMC designs appear to have a second-order sensitivity to tip/tilt modes, which is to say that the energy of the residual starlight in the final science plane grows as the amplitude of tip/tilt mode to the second power, for small tip/tilts. Figure 6 shows raw contrast of the residual light in the science plane, averaged between 2 and $8 \lambda / \mathrm{D}$ (in sky units), for different PIAACMC designs, and $0.004 \lambda / \mathrm{D}$ off-axis point source (thick curves) as well as on-axis point source (thin curves). The black and green curves represent ideal PIAACMC designs (each point on the Xaxis represents a different design). Black curve shows the case of an unobstructed pupil while green is the case of the $10 \%$ obstructed pupil. In both cases, an on-axis point source is fully suppressed, and the thin black and green curves would be identically 0 if there was no numerical computation error (as it is, the black thin curve is off the scale and thin green curve 
is $<10^{-10}$ contrast). Both curves exhibit the expected trend that coronagraphs with a larger IWA tend to be more tolerant to tip/tilt errors (and stellar angular sizes), except that for the case of the obstructed pupil, designs with IWAs between about 1.4 and 2.0 $\lambda / \mathrm{D}$ actually have the reverse trend. This tolerance can be improved by varying the remapped obstruction diameter at PIAA2, but is outside the scope of this paper. Based on this plot, however, we selected IWA = 1.4 as the point design to use for the design of manufacturable masks (in section 4).
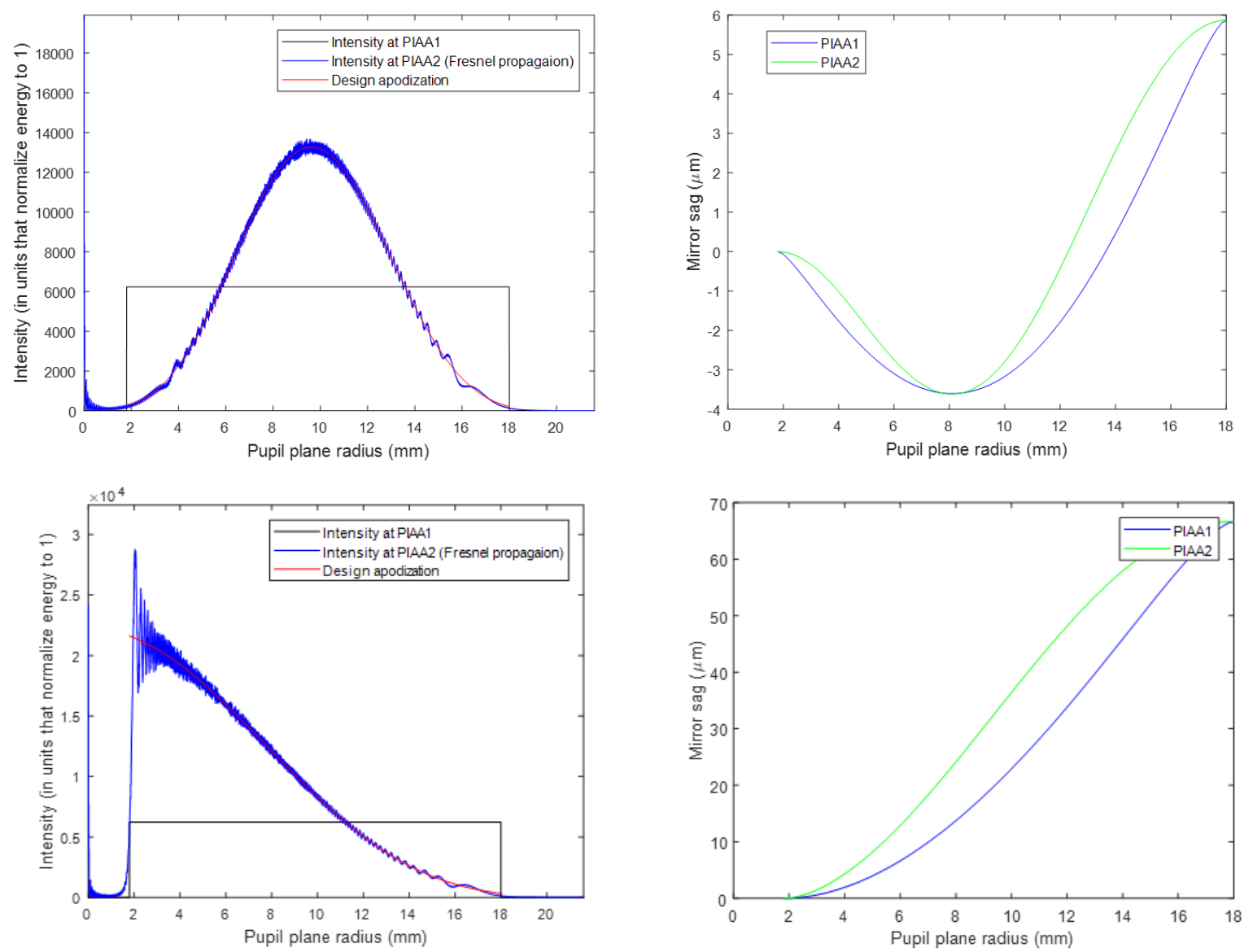

Figure 5. Examples of 1D PIAA apodizations and resulting PIAA mirror shapes. Top and bottom rows corresponds to PIAA apodizations for $q_{0}=2.6$, and $1.9 \mathrm{ND}$ in system units, respectively. Left column shows the original intensity at the pupil before PIAA, the design apodization, and the Fresnel-propagated apodization. The right column shows the PIAA mirror sags which reproduce the design apodization in the geometrical optics limit.

Although the green curve in Figure 6 (ideal PIAACMC design) shows good tolerance to tip/tilt, especially for IWA $=1.4$, the ideal CMC mask is challenging to manufacture because it requires an achromatic pi phase shift, and a mask radius that is proportional to wavelength. The blue and red curves represent more manufacturable masks (for ideal PIAA mirrors with a $10 \%$ obstructed pupil), which address the achromatic pi phase shift but not the size-scaling. The blue curve is an "ideal opaque mask", which is the same as the ideal CMC mask, except that it has 0 transmission instead of $-t$. It is a good solution for IWAs $>2 \lambda / \mathrm{D}$, where it converges to the ideal PIAACMC design because the ideal CMC mask transmission asymptotes to 0 as IWA grows. However, it has a poor performance for IWA $<2 \lambda / D$, where the partial transmission of the ideal CMC mask is needed for good performance. The red curve represents a mask which has a partially transmissive sag (optical path difference) which corresponds to a pi phase shift for the central wavelength. It is thus identical to the ideal CMC mask for the central wavelength, but imposes a slightly different phase shift for off-center wavelengths. It has much better performance than the ideal opaque mask, but still not as good as the ideal CMC mask. 


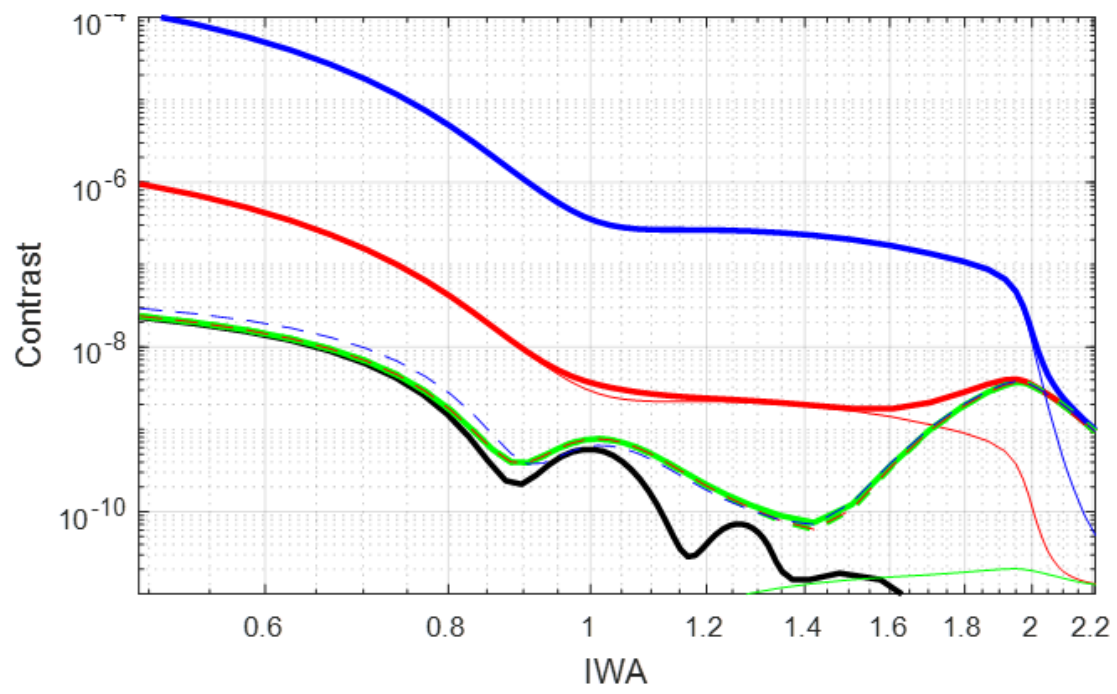

Figure 6. Average raw contrast between 2 and 8 ג/D on the sky for different ideal or quasi-ideal PIAACMC designs, in a 10\% band $(\Delta \lambda / \lambda)$. Every point on the $x$-axis represents a different PIAA apodization and PIAA mirror pair, parametrized by IWA. Black curve is the case of an unobstructed pupil and ideal CMC mask; colored curves are for a 10\% obstructed pupil and different masks: green is the ideal CMC, blue is the ideal opaque occulter, and red is the ideal monochromatic occulter. Thin curves represent the case of an on-axis point sources and thick lines represent an off-axis source at 0.004 N/D on sky.

The masks represented by the blue and red curves address the practical implementation of the achromatic pi phase shift, but not the scaling of the mask with wavelength. However, they do form interesting starting points for further mask design.

\section{FOCAL PLANE MASK}

We explored several design methodologies for a focal plane mask, as well as for different PIAA mirror designs and pupils. In this paper, we focus on the description of one of the methodologies and one of the designs. Specifically, we used a PIAA apodization from Section 3 for IWA $=1.4 \lambda / \mathrm{D}\left(\mathrm{q}_{0}=1.9\right)$, and $1 \mathrm{D}$ pupil apertures that have a $10 \%$ obstruction in the PIAA1, PIAA2, and Lyot stop planes. We then replaced the ideal PIAACMC mask by a "zone height mask" and used nonlinear optimization to solve for it.
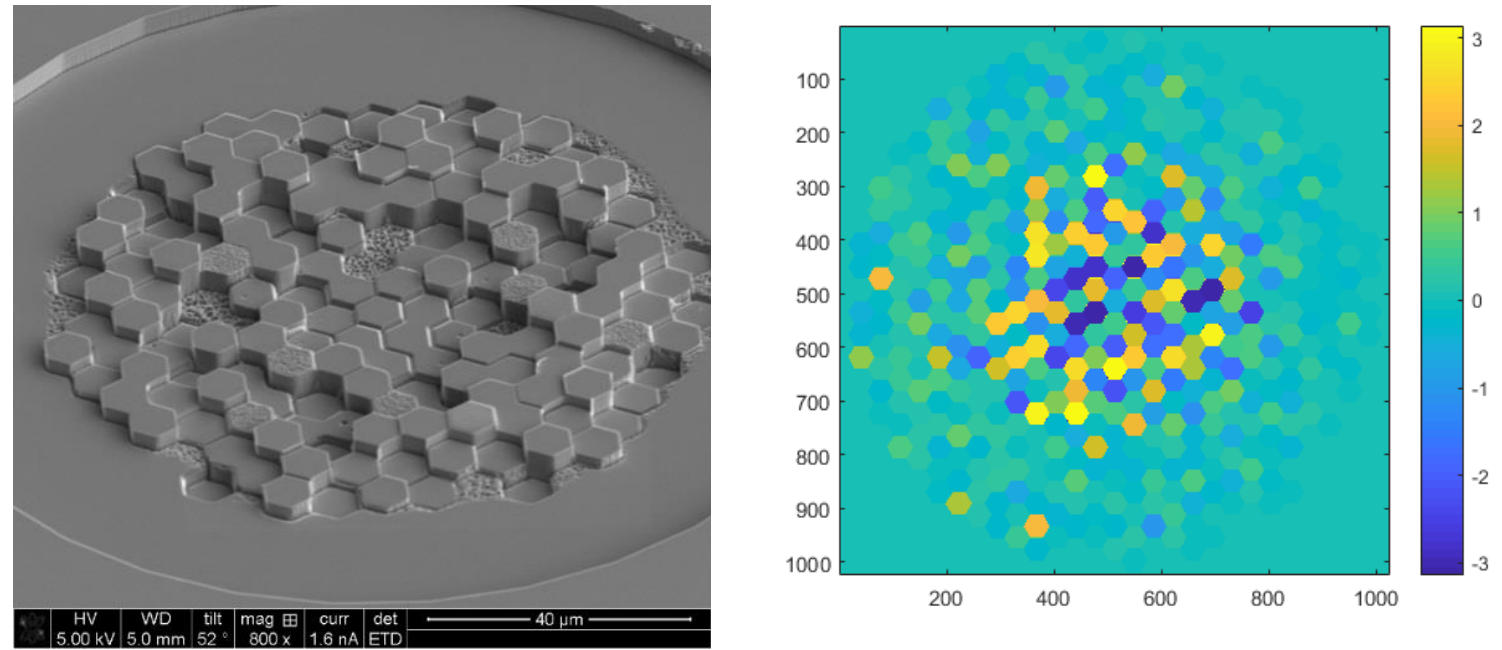

Figure 7. Left: example of a previously manufactured "zone height "mask (for the WFIRST PIAACMC design), showing heritage of the zone height masks. Right: one of our new design (not yet manufactured) for the LUVOIR pupil, which we call ZHM $v 1$. The units on the z-axis are radians of phase for the central wavelength. 
The zone height mask is similar to the one developed for the WFIRST PIAACMC design several years ago [6]. This mask was successfully manufactured and tested in vacuum, proving manufacturability and offering high maturity and heritage (see Figure 7, left). The mask is essentially a reflective hologram, or a mirror with a sag with a finely structured pattern, that consists of hexagonal zones at different heights.

To design the mask, we formulated an optimization problem and used a nonlinear optimization method called differential evolution to solve it, as part of a new coronagraph optimization package we are developing called csim. The optimization problem had a predefined optical layout (Figure 2), PIAA apodization as described above, and a predefined geometry of hexagonal zones (number of zones, their size, etc.). Its optimization variables were the heights (sags) of the hexagonal zones, and the objective function was the contrast between 2 and $8 \lambda / \mathrm{D}$ on the sky, in the presence of three off-axis sources, for a $10 \%$ band around $650 \mathrm{~nm}$. We let the optimization run until it achieved better than $10^{-9}$ contrast.

This produced a design that is now fully manufacturable, but achieves $10^{-9}$ contrast (between 2 and $8 \lambda / \mathrm{D}$ on the sky) with a pupil that has only a central obstruction (no struts or segments). Replacing this pupil by a LUVOIR pupil degrades the residual light in the science plane to $\sim 3 \times 10^{-6}$, but it turns out that this residual is correctable in broadband by a deformable mirror.

\section{ADDITIONAL SUPPRESSION BY DEFORMABLE MIRROR}

Deformable mirrors can also be the only coronagraphic component in the system, suppressing Airy rings as if they were speckles due to random errors. Used this way, they become equivalent to the "phase mask coronagraph", where the DM functions as a reconfigurable phase mask. However, it is challenging to achieve a high combination of throughput, bandwidth, inner working angle, and Strehl with a pure phase mask coronagraph. Therefore, for our design, we use the DM as a support element to remove the effects of struts and segments, rather than as a primary element.

Figure 8 shows the results of applying EFC to our PIAACMC design, which is the same as in Section 4, except we inserted the LUVOIR pupil, performed full diffraction propagations, and used conservative apertures and Lyot stop to reduce Fresnel effects, which reduced throughput from $100 \%$ for the ideal case to $74.9 \%$ (measured as the ratio of total energy between the entrance and exit pupils, for the case of no occulter). Some or even most of this throughput loss can potentially be recovered by using less conservative apertures, but we have not yet performed such a study and opted to simply use conservative stops.

The specifics of our EFC run were motivated primarily by modeling the test configuration and requirements of a lab demonstration that we are planning. Thus, our DM had $32 \times 32$ actuators, to match the $1 \mathrm{~K}$ device by Boston Micromachines, and our dark zone was single-sided rather than double-sided, testing a more conservative configuration consisting of 1DMs rather than 2DMs. (2DMs are usually needed to produce a double-sided dark zone, but that is outside the scope of this paper.) We also used a dark zone that has an inner edge of $2 \lambda / \mathrm{D}$, rather than $1.4 \lambda / \mathrm{D}$, per requirements of our laboratory demo. We have not yet explored more aggressive inner working angles with an EFC solution for this design, but no known limits to this exist, other than the $1.4 \lambda / \mathrm{D}$ (soft) limit imposed by the original coronagraph design.

The results in Figure 8 represent broadband simulations with a bandwidth of $10 \%$, and a dark zone that has an inner edge at 2 1/D and an outer diameter of 8 1/D (on the sky). For a point source, our contrast was $4.83 \times 10^{-10}$ (raw speckle intensity averaged across the dark zone), and for two point sources at tip and tilt of $4 \times 10^{-3} \lambda / \mathrm{D}$, the contrast was $8.6 \times 10^{-10}$. For a second order coronagraph, the contrast of a tipped or tilted point source also is approximately the same as the contrast for a Gaussian-distributed tip/tilt error, with a standard deviation equal to the off-axis displacement of the point source. So, the contrast values for individual off-axis sources can also be interpreted as contrast values for a Gaussian-distributed jitter with the root-mean-square value equal to the off-axis amount of the point source.

The surface of the deformable mirror which yielded these high contrast results is shown on the bottom of Figure 8 . The total sag is a less than $300 \mathrm{~nm}$, well within the stroke range of most deformable mirrors, and small enough that the Strehl ratio of planets is not degraded significantly (Strehl relative to the PSF of a flat DM was 0.83). In addition, we halted the EFC run before it asymptoted, so that deeper contrasts than what is shown in Figure 8 are possible. However, beyond a certain point, deeper contrasts with EFC come at the expense of greater DM strokes and worsened Strehl. 


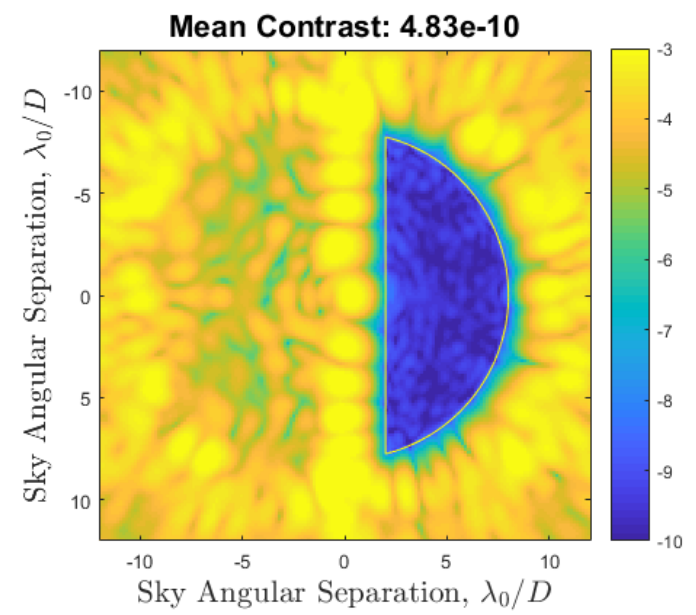

Tip\&Tilt: 4.0e-03, Mean Contrast: $8.60 \mathrm{e}-10$

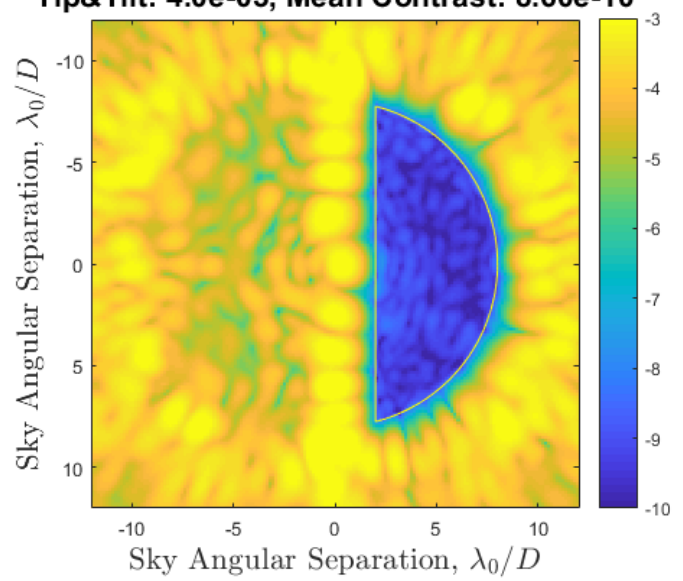

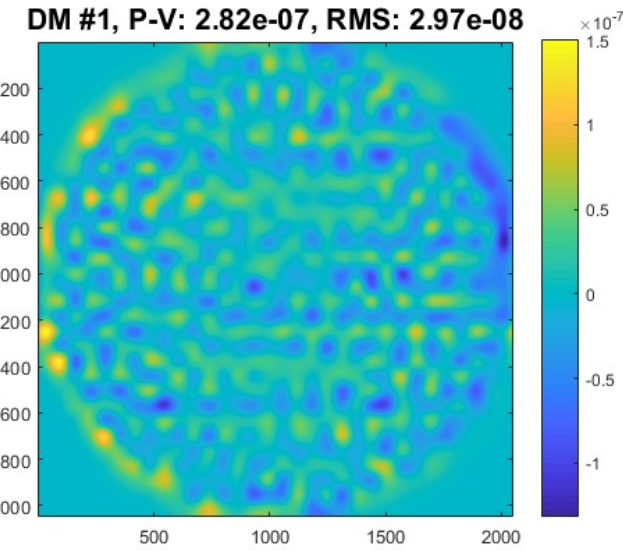

Figure 8. Top: Focal plane contrast in the focal plane with our PIAACMC design for the LUVOIR pupil. (Left: on-axis source only; Right: two off-axis sources at $4 \times 10^{-3} \lambda / D$ ). Bottom: the surface of our deformable mirror that generated these contrast maps. shown is the sag of the DM in units of meters.
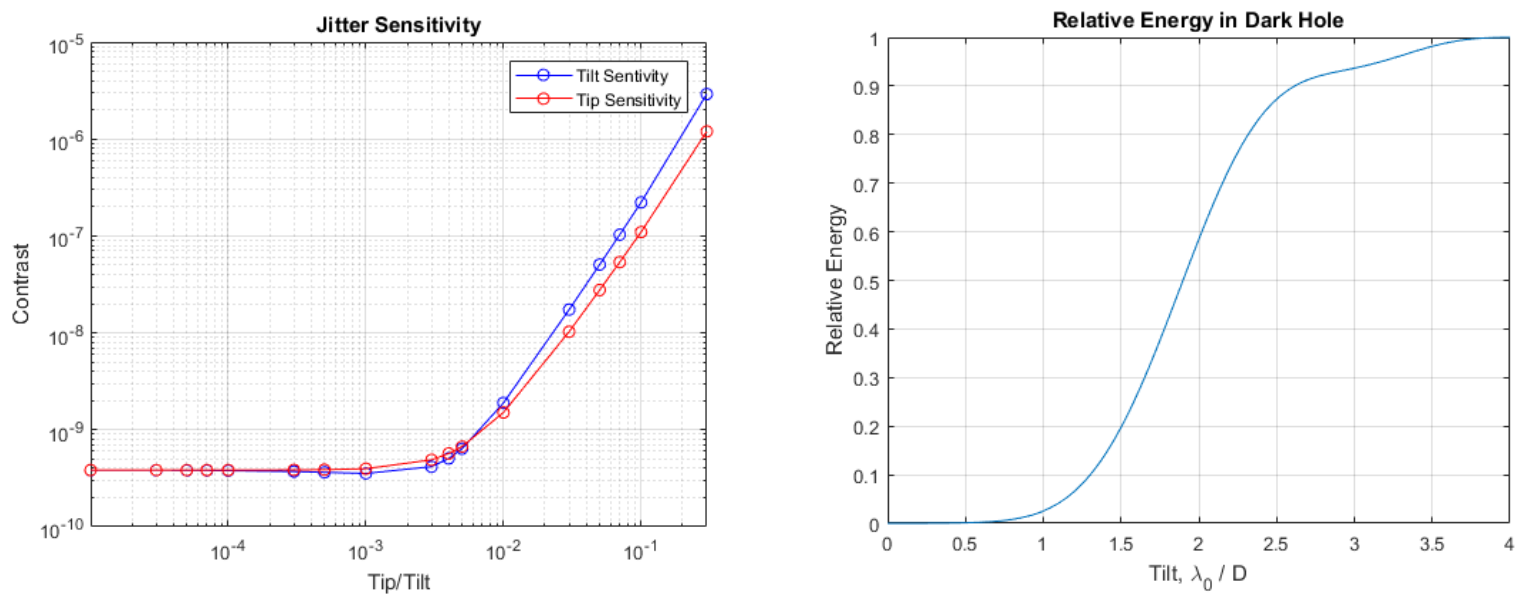

Figure 9. Left: Jitter sensitivity as a function of tip and tilt. Right: Total energy in the dark zone, normalized to the middle of the dark zone. We define the inner working angle as the 50\% point of this curve, which is 1.8 ND. 


\section{CONCLUSIONS}

The PIAACMC coronagraph architecture is attractive because it can achieve very high throughput and inner working angles, which can enable large savings on mission cost (by enabling the same science with a smaller aperture), or significantly increased science at the same cost point.

We have produced a preliminary manufacturable PIAACMC design for the LUVOIR pupil, which achieves better than $10^{-9}$ contrast between 2 and $8 \lambda / \mathrm{D}$, in a $10 \%$ band, under point source tip and tilts of $0.004 \lambda / \mathrm{D}$, as well as Gaussian jitter with the same rms. The total throughput (ratio of energies between entrance and exit pupil in the case of no occulter) is $\sim 75 \%$. We have only explored a small volume of the trade space, and it is possible that all of these parameters can be further improved.

Our design relied on the deformable mirror to remove the light residual caused by the pupil struts and segments. This simplified the design of the PIAACMC coronagraph from 2D to 1D, and provides an opportunity for faster turnaround of designs and more effective trade space studies in the future. This technique has a price of requiring more stroke on the deformable mirror and degrading Strehl, but for the case of the LUVOIR pupil, these costs remain relatively benign.

\section{ACKNOWLEDGEMENTS}

This work was supported in part by the National Aeronautics and Space Administration's Ames Research Center, as well as the NASA Strategic Astrophysics Technology - Technology Development for Exoplanet Missions (SAT-TDEM) program through solicitation NNH16ZDA001N-SAT at NASA's Science Mission Directorate. It was carried out at the NASA Ames Research Center, the NASA Jet Propulsion Laboratory, and the University of Arizona. Any opinions, findings, and conclusions or recommendations expressed in this article are those of the authors and do not necessarily reflect the views of the National Aeronautics and Space Administration.

\section{REFERENCES}

[1] Guyon, O., Martinache, F., Belikov, R., Soummer, R., "High performance PIAA coronagraphy with complex amplitude focal plane masks," ApJS 190, 2, pp. 220-232 (2010).

[2] Guyon, O., Hinz, P.M., Cady, E., Belikov, R., Martinache, F. 2014, "High Performance Lyot and PIAA Coronagraphy for Arbitrarily Shaped Telescope Apertures," ApJ 780, 171.

[3] Stark, C.C. et al. 2015, "Lower Limits on Aperture Size for an ExoEarth Detecting Coronagraphic Mission," ApJ 808, 149.

[4] Soummer , R., "Apodized Pupil Lyot Coronagraphs for arbitrary telescope apertures," ApJL, 618 (2004).

[5] Belikov R., Kasdin N.J., Vanderbei R.J., "Diffraction-based Sensitivity Analysis of Apodized Pupil-Mapping Systems," ApJ Vol 652, issue 1, pp. 833-844, 11/2006.

[6] Kern, B.D., Wilson, D. W., Guyon, O., Muller, R. E., Balasubramanian, K., Sidick, E., Poberezhskiy, I., Belikov, R., "PIAACMC coronagraphic occulting mask fabrication, characterization, and modeling," Proc SPIE 9605-11, (2015). 